\title{
On Stable Hypersurfaces with Vanishing Scalar Curvature
}

\author{
Gregório Silva Neto
}

May, 242013

\begin{abstract}
We will prove that there are no stable complete hypersurfaces of $\mathbb{R}^{4}$ with zero scalar curvature, polynomial volume growth and such that $\frac{(-K)}{H^{3}} \geq c>0$ everywhere, for some constant $c>0$, where $K$ denotes the Gauss-Kronecker curvature and $H$ denotes the mean curvature of the immersion. Our second result is the Bernstein type one there is no entire graphs of $\mathbb{R}^{4}$ with zero scalar curvature such that $\frac{(-K)}{H^{3}} \geq c>0$ everywhere. At last, it will be proved that, if there exists a stable hypersurface with zero scalar curvature and $\frac{(-K)}{H^{3}} \geq c>0$ everywhere, that is, with volume growth greater than polynomial, then its tubular neighborhood is not embedded for suitable radius.
\end{abstract}

\section{Introduction}

Let $x: M^{3} \rightarrow \mathbb{R}^{4}$ be an isometric immersion. If $\lambda_{1}, \lambda_{2}, \lambda_{3}$ are the eigenvalues of the second fundamental form, then the scalar curvature $R$, the non-normalized mean curvature $H$, and the Gauss-Kronecker curvature $K$ are given, respectively, by

$$
R=\lambda_{1} \lambda_{2}+\lambda_{1} \lambda_{3}+\lambda_{2} \lambda_{3}, \quad H=\lambda_{1}+\lambda_{2}+\lambda_{3} \text { and } K=\lambda_{1} \lambda_{2} \lambda_{3} .
$$


In 1959, Hartman and Nirenberg, cf. [8], have shown that the only surfaces with zero Gaussian curvature in three-dimensional Euclidean space are planes and cylinders.

Generalizing this fact, in 1977, Cheng and Yau, cf. [16], showed that the only complete non-compact hypersurfaces with constant scalar curvature and non-negative sectional curvature in the Euclidean space $\mathbb{R}^{n+1}$ are the generalized cylinders $\mathbb{S}^{n-p} \times \mathbb{R}^{p}$.

Let $D \subset M^{3}$ be a regular domain, i.e., a domain with compact closure and piecewise smooth boundary. A compact supported variation of the immersion $x$ is a differentiable map $X:(-\varepsilon, \varepsilon) \times D \rightarrow \mathbb{R}^{4}, \varepsilon>0$, such that, for each $t \in(-\varepsilon, \varepsilon), X_{t}: D \rightarrow \mathbb{R}^{4}, X_{t}(p)=$ $X(t, p)$ is an immersion, $X_{0}=\left.x\right|_{D}$ and $\left.X_{t}\right|_{\partial D}=\left.X_{0}\right|_{\partial D}$. We recall that hypersurfaces of $\mathbb{R}^{4}$ with zero scalar curvature are critical points of the functional

$$
\mathcal{A}_{1}(t)=\int_{M} H(t) d M_{t}
$$

under all variations compactly supported in $D$ (see [13], [1], [14], 44).

Following Alencar, do Carmo, and Elbert, cf. [2], let us define the concept of stability for immersions with zero scalar curvature. Let $A: T M \rightarrow T M$ the linear operator associated to the second fundamental form of immersion $x$. We define the first Newton transformation $P_{1}: T M \rightarrow T M$ by $P_{1}=H I-A$, where $I$ denotes identity operator. We now introduce a second order differential operator which will play a role similar to that of Laplacian in the minimal case:

$$
L_{1}(f)=\operatorname{div}\left(P_{1}(\nabla f)\right)
$$

where $\operatorname{div} X$ denotes the divergence of vector field $\mathrm{X}$, and $\nabla f$ denotes the gradient of the function $f$ in the induced metric. In [9], Hounie and Leite showed that $L_{1}$ is elliptic if and only if rank $A>1$. Thus, $K \neq 0$ everywhere implies $L_{1}$ is elliptic, and if $H>0$, then $P_{1}$ is a positive definite linear operator.

Computing the second derivative of functional $\mathcal{A}_{1}$ we obtain

$$
\left.\frac{d^{2} \mathcal{A}_{1}}{d t^{2}}\right|_{t=0}=-2 \int_{M} f\left(L_{1} f-3 K f\right) d M,
$$

where $f=\left\langle\frac{d X}{d t}(0), \eta\right\rangle$, and $\eta$ is the normal vector field of the immersion. 
Since $H^{2}=|A|^{2}+2 R$, if $R=0$ then $H^{2}=|A|^{2}$, i.e, if $K \neq 0$ everywhere, then $H^{2}=|A|^{2} \neq 0$ everywhere. It implies that $H>0$ everywhere or $H<0$ everywhere. Hence, unlike minimal case, the sign of functional $\mathcal{A}_{1}$ depends on choice of orientation of $M^{3}$. Following Alencar, do Carmo and Elbert, see [2], if we choose an orientation such that $H>0$ everywhere, then the immersion will be stable if $\left.\frac{d^{2} \mathcal{A}_{1}}{d t^{2}}\right|_{t=0}>0$ under all compact support variations. Otherwise, i. e., if we choose an orientation such that $H<0$, then $x$ is stable if $\left.\frac{d^{2} \mathcal{A}_{1}}{d t^{2}}\right|_{t=0}<0$. For more details, see [2].

In the pursuit of this subject, Alencar, do Carmo and Elbert, cf. [2], have posed the following:

Question. Is there any stable complete hypersurface $M^{3}$ in $\mathbb{R}^{4}$ with zero scalar curvature and everywhere non-zero Gauss-Kronecker curvature?

The goal of this paper is to give some partial answers to this question. Let $B_{r}(p)$ be the geodesic ball with center $p \in M$ and radius $r$. We say that a Riemannian manifold $M^{3}$ has polynomial volume growth, if there exists $\alpha \in[0,4]$ such that

$$
\frac{\operatorname{vol}\left(B_{r}(p)\right)}{r^{\alpha}}<\infty
$$

for all $p \in M$.

A well known inequality establishes that

$$
H K \leq \frac{1}{2} R^{2}
$$

If $R=0$ and $K \neq 0$ everywhere, then the quotient $\frac{K}{H^{3}}$ is always negative, independent on choice of orientation. Furthermore, considering $K$ and $H^{3}$ as functions of the eigenvalues of second fundamental form, we can see that

$$
0<\frac{(-K)}{H^{3}} \leq \frac{4}{27}
$$

provided $K$ and $H^{3}$ are homogeneous polynomials of degree 3. For details, see Appendix.

The first result is 
Theorem A. There is no stable complete hypersurface $M^{3}$ of $\mathbb{R}^{4}$ with zero scalar curvature, polynomial volume growth and such that

$$
\frac{(-K)}{H^{3}} \geq c>0
$$

everywhere, for some constant $c>0$. Here $H$ denotes the mean curvature and $K$ denotes the Gauss-Kronecker curvature of the immersion.

As a consequence of Theorem A, we obtain the following Bernstein type result.

Theorem B. There are no entire graphs $M^{3}$ of $\mathbb{R}^{4}$ with zero scalar curvature and such that

$$
\frac{(-K)}{H^{3}} \geq c>0
$$

everywhere, for some constant $c>0$. Here $H$ denotes the mean curvature and $K$ denotes the Gauss-Kronecker curvature of the immersion.

Following Nelli and Soret, cf. [12, in section 5 we show that, if $M^{3}$ is a stable complete hypersurface of $\mathbb{R}^{4}$ with zero scalar curvature and such that $\frac{(-K)}{H^{3}} \geq c>0$ everywhere, then the tube around $M$ is not embedded for suitable radius. Precisely, we define the tube of radius $h$ around $M$ the set

$$
T(M, h)=\left\{x \in \mathbb{R}^{4} ; \exists p \in M, x=p+t \eta, t \leq h(p)\right\}
$$

where $\eta$ is the normal vector of second fundamental form of the immersion and $h: M \rightarrow \mathbb{R}$ is an everywhere non-zero smooth function. We prove

Theorem C. Let $M^{3}$ be a stable complete hypersurface of $\mathbb{R}^{4}$ with vanishing scalar curvature. Suppose that the second fundamental form of the immersion is bounded and there exists a constant $c>0$ such that $\frac{(-K)}{H^{3}} \geq c>0$ everywhere. Then, for constants $0<b_{1} \leq 1, b_{2}>0$, and for any smooth function $h: M \rightarrow \mathbb{R}$ satisfying

$$
h(p) \geq \min \left\{\frac{b_{1}}{|A(p)|}, b_{2} \rho(p)^{\delta}\right\}, p \in M, \delta>0,
$$

the tube $T(M, h)$ is not embedded. Here, $\rho(p)$ denotes the intrinsic distance in $M$ to a fixed point $p_{0} \in M$. 
Acknowledgements. I would like to thank professor Hilário Alencar for read critically this manuscript and for his many valuable suggestions. I would also to thank Detang Zhou by his useful suggestions, and professor Barbara Nelli for her suggestions to clarify some arguments used in the proof of Theorem C.

\section{Preliminary Results}

Let $B(X, Y)=\bar{\nabla}_{X} Y-\nabla_{X} Y$ be the second fundamental form of immersion $x$, where $\nabla$ and $\bar{\nabla}$ are the connections of $M^{3}$ and $\mathbb{R}^{4}$, respectively. The shape operator is the only symmetric linear operator $A: T M \rightarrow T M$ such that

$$
B(X, Y)=\langle A(X), Y\rangle \eta, \forall X, Y \in T M
$$

where $\eta$ is the normal field of the immersion $x$.

Denote by $|A|^{2}=\operatorname{tr}\left(A^{2}\right)$ the matrix norm of second fundamental form. Since $H^{2}=$ $|A|^{2}+2 R$, if $R=0$, then $H^{2}=|A|^{2}$. Hence, $K \neq 0$ everywhere implies $H=|A| \neq 0$ everywhere, and we can choose an orientation of $M$ such that $H>0$ everywhere.

Remark 2.1. From now on, let us fix an orientation of $M^{3}$ such that $H>0$ everywhere.

A well known inequality establishes that

$$
H K \leq \frac{1}{2} R^{2}
$$

Therefore, by using inequality above, $R=0$ and $H>0$ everywhere implies $K<0$ everywhere.

Define $P_{1}: T M \rightarrow T M$ by $P_{1}=H I-A$ the first Newton transformation. If $R=0$ and $H>0$, then $P_{1}$ is positive definite. It was proved by Hounie and Leite in a general point of view, see [9]. In fact, $P_{1}$ positive definite implies $L_{1}(f)=\operatorname{div}\left(P_{1}(\nabla f)\right)$ is an elliptic differential operator. Let us give here a proof for sake of completeness. It suffices to prove that $H-\lambda_{i}>0, i=1,2,3$. In fact,

$$
\lambda_{1}^{2}\left(H-\lambda_{1}\right)=\lambda_{1}^{2}\left(\lambda_{2}+\lambda_{3}\right)=\lambda_{1}^{2} \lambda_{2}+\lambda_{1}^{2} \lambda_{3}
$$


Since $R=\lambda_{1} \lambda_{2}+\lambda_{1} \lambda_{3}+\lambda_{2} \lambda_{3}=0$, we have

$$
0=\lambda_{1} R=\lambda_{1}\left(\lambda_{1} \lambda_{2}+\lambda_{1} \lambda_{3}+\lambda_{2} \lambda_{3}\right)=\lambda_{1}^{2} \lambda_{2}+\lambda_{1}^{2} \lambda_{3}+\lambda_{1} \lambda_{2} \lambda_{3}
$$

i.e.,

$$
\lambda_{1}^{2} \lambda_{2}+\lambda_{1}^{2} \lambda_{3}=-\lambda_{1} \lambda_{2} \lambda_{3}=-K>0
$$

Thus,

$$
\lambda_{1}^{2}\left(H-\lambda_{1}\right)=\lambda_{1}^{2} \lambda_{2}+\lambda_{1}^{2} \lambda_{3}=-\lambda_{1} \lambda_{2} \lambda_{3}>0,
$$

and then, $H-\lambda_{1}>0$. The other cases are analogous.

Our choice of orientation, i.e, that one such that $H>0$ everywhere, implies stability condition is equivalent to

$$
-3 \int_{M} K f^{2} d M \leq \int_{M}\left\langle P_{1}(\nabla f), \nabla f\right\rangle d M
$$

The inequality (2.1) is known as stability inequality.

Remark 2.2. When $H<0$, then $K>0$ and $P_{1}$ is negative definite. In this case, stability condition is equivalent to

$$
3 \int_{M} K f^{2} d M \leq \int_{M}\left\langle\left(-P_{1}\right)(\nabla f), \nabla f\right\rangle d M
$$

Let $\nabla A(X, Y, Z):=\left\langle\nabla_{Z}(A(X))-A\left(\nabla_{Z} X\right), Y\right\rangle$ be the covariant derivative of operator $A$. The following proposition will play an important role in the proof of main theorems. In [6], do Carmo and Peng showed a very similar inequality for minimal hypersurfaces.

Proposition 2.1. If $R=0$ and there exists $c>0$ such that $\frac{-K}{H^{3}} \geq c>0$ everywhere, then there exists $c_{0}>0$, depending on $c$, such that

$$
|\nabla A|^{2}-|\nabla H|^{2} \geq \frac{2}{1+2 c_{0}^{2}}|\nabla H|^{2},
$$

where $\nabla H$ denotes the gradient of $H$. 
Proof. Let us fix $p \in M$ and choose $\left\{e_{1}(p), e_{2}(p), e_{3}(p)\right\}$ an orthonormal basis of $T_{p} M$ such that $h_{i j}(p)=\lambda_{i}(p) \delta_{i j}$, where $h_{i j}=\left\langle A\left(e_{i}\right), e_{j}\right\rangle, \lambda_{i}(p)$ denotes the eigenvalues of $A$ in $p$ and $\delta_{i j}$ is the Kronecker delta

$$
\delta_{i j}=\left\{\begin{array}{lll}
1 & \text { if } & i=j \\
0 & \text { if } & i \neq j
\end{array}\right.
$$

Extending this basis by parallel transport along geodesics starting on $p$, to a referential in a neighbourhood of $p$, we have $\nabla_{e_{i}(p)} e_{j}(p)=0$, for all $i, j=1,2,3$. This is called geodesic referential at $p$.

Let us denote by $h_{i j ; k}=\left(h_{i j}\right)_{k}:=e_{k}\left(h_{i j}\right)$ the covariant derivatives of function $h_{i j}$, and by $h_{i j k}$ the components of tensor $\nabla A$ in the referential $\left\{e_{1}, e_{2}, e_{3}\right\}$, i.e., $h_{i j k}=$ $\nabla A\left(e_{i}, e_{j}, e_{k}\right)$. Since $\left\{e_{1}, e_{2}, e_{3}\right\}$ is a geodesic referential, we have

$$
\begin{aligned}
h_{i j k} & =\nabla A\left(e_{i}, e_{j}, e_{k}\right)=\left\langle\nabla_{e_{k}}\left(A\left(e_{i}\right)\right)-A\left(\nabla_{e_{k}} e_{i}\right), e_{j}\right\rangle=\left\langle\nabla_{e_{k}}\left(A\left(e_{i}\right)\right), e_{j}\right\rangle \\
& =e_{k}\left(\left\langle A\left(e_{i}\right), e_{j}\right\rangle\right)-\left\langle A\left(e_{i}\right), \nabla_{e_{k}} e_{j}\right\rangle=e_{k}\left(\left\langle A\left(e_{i}\right), e_{j}\right\rangle\right)=e_{k}\left(h_{i j}\right) \\
& =h_{i j ; k} .
\end{aligned}
$$

Since $R=0$, then $H^{2}=|A|^{2}$. Using this fact, we have

$$
\begin{aligned}
4 H^{2}|\nabla H|^{2} & =\left|\nabla\left(H^{2}\right)\right|^{2}=\left|\nabla\left(|A|^{2}\right)\right|^{2}=\sum_{k=1}^{3}\left[\left(\sum_{i, j=1}^{3} h_{i j}^{2}\right)_{k}\right]^{2} \\
& =\sum_{k=1}^{3}\left(\sum_{i, j=1}^{3} 2 h_{i j} h_{i j ; k}\right)^{2}=4 \sum_{k=1}^{3}\left(\sum_{i=1}^{3} h_{i i} h_{i i ; k}\right)^{2} .
\end{aligned}
$$

Now, using Cauchy-Schwarz inequality, we obtain

$$
\begin{aligned}
4 \sum_{k=1}^{3}\left(\sum_{i=1}^{3} h_{i i} h_{i i ; k}\right)^{2} & \leq 4 \sum_{k=1}^{3}\left[\left(\sum_{i=1}^{3} h_{i i}^{2}\right)\left(\sum_{i=1}^{3} h_{i i ; k}^{2}\right)\right] \\
& =4|A|^{2}\left(\sum_{i, k=1}^{3} h_{i i ; k}^{2}\right)=4 H^{2}\left(\sum_{i, k=1}^{3} h_{i i ; k}^{2}\right) .
\end{aligned}
$$


Therefore,

$$
|\nabla H|^{2} \leq \sum_{i, k=1}^{3} h_{i i ; k}^{2} .
$$

On the other hand, since $R=h_{11} h_{22}+h_{11} h_{33}+h_{22} h_{33}-h_{12}^{2}-h_{13}^{2}-h_{23}^{2}=0$, we have for $k=1,2,3$,

$$
\begin{aligned}
0= & \left(h_{11} h_{22}+h_{11} h_{33}+h_{22} h_{33}-h_{12}^{2}-h_{13}^{2}-h_{23}^{2}\right)_{k} \\
= & h_{11 k} h_{22}+h_{11} h_{22 k}+h_{11 k} h_{33}+h_{11} h_{33 k}+h_{22 k} h_{33}+h_{22} h_{33 k} \\
& \quad-2 h_{12} h_{12 k}-2 h_{13} h_{13 k}-2 h_{23} h_{23 k} \\
= & h_{11 k} h_{22}+h_{11} h_{22 k}+h_{11 k} h_{33}+h_{11} h_{33 k}+h_{22 k} h_{33}+h_{22} h_{33 k} \\
= & h_{11 k}\left(h_{22}+h_{33}\right)+h_{22 k}\left(h_{11}+h_{33}\right)+h_{33 k}\left(h_{11}+h_{22}\right) \\
= & h_{11 k}\left(H-h_{11}\right)+h_{22 k}\left(H-h_{22}\right)+h_{33 k}\left(H-h_{33}\right)
\end{aligned}
$$

Thus, taking $k=1$ in the inequality above, we have

$$
h_{111}=-\frac{1}{H-h_{11}}\left[h_{221}\left(H-h_{22}\right)+h_{331}\left(H-h_{33}\right)\right]
$$

Analogously, taking $k=2$ and $k=3$ we have

$$
h_{222}=-\frac{1}{H-h_{22}}\left[h_{112}\left(H-h_{11}\right)+h_{332}\left(H-h_{33}\right)\right]
$$

and

$$
h_{333}=-\frac{1}{H-h_{33}}\left[h_{113}\left(H-h_{11}\right)+h_{223}\left(H-h_{22}\right)\right] .
$$

Squaring and summing, we have

$$
\begin{aligned}
h_{111}^{2}+h_{222}^{2}+h_{333}^{2}= & \frac{1}{\left(H-h_{11}\right)^{2}}\left[h_{221}\left(H-h_{22}\right)+h_{331}\left(H-h_{33}\right)\right]^{2} \\
& \frac{1}{\left(H-h_{22}\right)^{2}}\left[h_{112}\left(H-h_{22}\right)+h_{332}\left(H-h_{33}\right)\right]^{2} \\
& \frac{1}{\left(H-h_{33}\right)^{2}}\left[h_{113}\left(H-h_{11}\right)+h_{223}\left(H-h_{22}\right)\right]^{2}
\end{aligned}
$$


Using inequality $(a+b)^{2}=a^{2}+2 a b+b^{2} \leq 2\left(a^{2}+b^{2}\right)$, we have

$$
\begin{aligned}
h_{111}^{2}+h_{222}^{2}+h_{333}^{2} \leq 2 & {\left[\left(\frac{H-h_{22}}{H-h_{11}}\right)^{2} h_{221}^{2}+\left(\frac{H-h_{33}}{H-h_{11}}\right)^{2} h_{331}^{2}\right.} \\
& \left(\frac{H-h_{11}}{H-h_{22}}\right)^{2} h_{112}^{2}+\left(\frac{H-h_{33}}{H-h_{22}}\right)^{2} h_{332}^{2} \\
& \left.\left(\frac{H-h_{11}}{H-h_{33}}\right)^{2} h_{113}^{2}+\left(\frac{H-h_{22}}{H-h_{33}}\right)^{2} h_{223}^{2}\right]
\end{aligned}
$$

Since the functions

$$
g_{i j}: \mathbb{R}^{3} \rightarrow \mathbb{R}, g_{i j}\left(h_{11}, h_{22}, h_{33}\right)=\left(\frac{H-h_{i i}}{H-h_{j j}}\right)^{2}, i, j=1,2,3
$$

are quotients of homogeneous polynomials of same degree, the values of $g_{i j}$ depends only on its value in the unit sphere $\mathbb{S}^{2}$. Since $\left\{\left(h_{11}, h_{22}, h_{33}\right) \in \mathbb{R}^{3} \mid R=0\right\}$ is closed in $\mathbb{R}^{3}$, $\left\{\left(h_{11}, h_{22}, h_{33}\right) \in \mathbb{R}^{3} \mid \frac{-K}{H^{3}} \geq c>0\right\}=\left\{\left(h_{11}, h_{22}, h_{33}\right) \in \mathbb{S}^{2} \mid \frac{-K}{H^{3}} \geq c>0\right\}$ and $\mathbb{S}^{2}$ are compact sets of $\mathbb{R}^{3}$, their intersection is a compact set of $\mathbb{S}^{2}$. Thus all the functions $g_{i j}$ has a maximum and a minimum in $\mathbb{S}^{2}$. Let $c_{0}>0$ the maximum of the maxima of the functions $g_{i j}, i, j=1,2,3$. Then

$$
h_{111}^{2}+h_{222}^{2}+h_{333}^{2} \leq 2 c_{0}^{2}\left(h_{112}^{2}+h_{113}^{2}+h_{221}^{2}+h_{223}^{2}+h_{331}^{2}+h_{332}^{2}\right) .
$$

This implies

$$
\begin{aligned}
|\nabla H|^{2} \leq & \sum_{i, k=1}^{3} h_{i i k}^{2}=h_{111}^{2}+h_{112}^{2}+h_{113}^{2}+h_{221}^{2}+h_{222}^{2}+h_{223}^{2} \\
& \quad+h_{331}^{2}+h_{331}^{2}+h_{333}^{2} \\
\leq & \left(1+2 c_{0}^{2}\right)\left(h_{112}^{2}+h_{113}^{2}+h_{221}^{2}+h_{223}^{2}+h_{331}^{2}+h_{332}^{2}\right) \\
\leq & \left(1+2 c_{0}^{2}\right)\left[\frac{1}{2}\left(h_{121}^{2}+h_{211}^{2}\right)+\frac{1}{2}\left(h_{131}^{2}+h_{311}^{2}\right)+\frac{1}{2}\left(h_{212}^{2}+h_{122}^{2}\right)\right. \\
& \left.\frac{1}{2}\left(h_{232}^{2}+h_{322}^{2}\right)+\frac{1}{2}\left(h_{313}^{2}+h_{133}^{2}\right)+\frac{1}{2}\left(h_{323}^{2}+h_{223}^{2}\right)\right] \\
= & \frac{1+2 c_{0}^{2}}{2}\left(h_{121}^{2}+h_{211}^{2}+h_{131}^{2}+h_{311}^{2}+h_{212}^{2}+h_{122}^{2}\right. \\
& \left.+h_{232}^{2}+h_{322}^{2}+h_{313}^{2}+h_{133}^{2}+h_{323}^{2}+h_{233}^{2}\right) .
\end{aligned}
$$


Therefore,

$$
\begin{aligned}
|\nabla A|^{2}=\sum_{i, j, k=1}^{3} h_{i j k}^{2} & \geq \sum_{i, k=1}^{3} h_{i i k}^{2}+\sum_{i \neq k=1}^{3} h_{i k i}^{2}+\sum_{i \neq k=1}^{3} h_{k i i}^{2} \\
& \geq|\nabla H|^{2}+\frac{2}{1+2 c_{0}^{2}}|\nabla H|^{2} \\
& =\left(1+\frac{2}{1+2 c_{0}^{2}}\right)|\nabla H|^{2} .
\end{aligned}
$$

\section{Main Theorems}

Hereafter, we will fix a point $p_{0} \in M$ and denote by $B_{r}$ the geodesic (intrinsic) ball of center $p_{0}$ and radius $r$.

The main tool to prove Theorem A stated in the Introduction is the following

Proposition 3.1. Let $x: M^{3} \rightarrow \mathbb{R}^{4}$ be a stable isometric immersion with zero scalar curvature and such that $K$ is nowhere zero. Then, for all smooth function $\psi$ with compact support in $M$, for all $\delta>0$ and $0<q<\sqrt{\frac{2}{1+2 c_{0}^{2}}}$, there exists constants $\Lambda_{1}(q), \Lambda_{2}(q)>0$ such that

$$
\int_{M} H^{5+2 q}\left(\frac{(-K)}{H^{3}}-\Lambda_{1} \delta^{\frac{5+2 q}{3+2 q}}\right) \psi^{5+2 q} d M \leq \Lambda_{2} \delta^{-\frac{5+2 q}{2}} \int_{M}|\nabla \psi|^{5+2 q} d M
$$

Proof. Let us choose an orientation such that $H>0$ and apply the corresponding stability inequality

$$
3 \int_{M}(-K) f^{2} d M \leq \int_{M}\left\langle P_{1}(\nabla f), \nabla f\right\rangle d M
$$

for $f=H^{1+q} \varphi$, where $q>0$, and $\varphi$ is a smooth function compactly supported on $M$. First note that

$$
\nabla f=\nabla\left(H^{1+q} \varphi\right)=(1+q) H^{q} \varphi \nabla H+H^{1+q} \nabla \varphi
$$


It implies

$$
\begin{aligned}
\left\langle P_{1}(\nabla f), \nabla f\right\rangle= & (1+q)^{2} H^{2 q} \varphi^{2}\left\langle P_{1}(\nabla H), \nabla H\right\rangle \\
& +2(1+q) H^{1+2 q} \varphi\left\langle P_{1}(\nabla H), \nabla \varphi\right\rangle \\
& +H^{2+2 q}\left\langle P_{1}(\nabla \varphi), \nabla \varphi\right\rangle .
\end{aligned}
$$

Since $H>0$, then $P_{1}$ is positive definite. Now, let us estimate the second term in the right hand side of identity above. By using Cauchy-Schwarz inequality followed by inequality $x y \leq \frac{x^{2}}{2}+\frac{y^{2}}{2}$, for all $x, y \in \mathbb{R}$, we obtain

$$
\begin{aligned}
H^{1+2 q} \varphi\left\langle P_{1}(\nabla H), \nabla \varphi\right\rangle & =H^{2 q}\left\langle\sqrt{\beta} \varphi \sqrt{P_{1}}(\nabla H),(1 / \sqrt{\beta}) H \sqrt{P_{1}}(\nabla \varphi)\right\rangle \\
& \leq H^{2 q}\left\|\sqrt{\beta} \varphi \sqrt{P_{1}}(\nabla H)\right\|\left\|(1 / \sqrt{\beta}) H \sqrt{P_{1}}(\nabla \varphi)\right\| \\
& \leq H^{2 q}\left(\frac{\left\|\sqrt{\beta} \varphi \sqrt{P_{1}}(\nabla H)\right\|^{2}}{2}+\frac{\left\|(1 / \sqrt{\beta}) H \sqrt{P_{1}}(\nabla \varphi)\right\|^{2}}{2}\right) \\
& =\frac{\beta}{2} H^{2 q} \varphi^{2}\left\langle P_{1}(\nabla H), \nabla H\right\rangle+\frac{1}{2 \beta} H^{2+2 q}\left\langle P_{1}(\nabla \varphi), \nabla \varphi\right\rangle,
\end{aligned}
$$

for any constant $\beta>0$. Then stability inequality $(3.2)$ becomes

$$
\begin{aligned}
3 \int_{M}(-K) H^{2+2 q} \varphi^{2} d M \leq & (1+q)^{2} \int_{M} H^{2 q} \varphi^{2}\left\langle P_{1}(\nabla H), \nabla H\right\rangle d M \\
& +2(1+q) \int_{M} H^{1+2 q} \varphi\left\langle P_{1}(\nabla H), \nabla \varphi\right\rangle d M \\
& +\int_{M} H^{2+2 q}\left\langle P_{1}(\nabla \varphi), \nabla \varphi\right\rangle d M \\
\leq & \left((1+q)^{2}+(1+q) \beta\right) \int_{M} H^{2 q} \varphi^{2}\left\langle P_{1}(\nabla H), \nabla H\right\rangle d M \\
& +\left(1+\frac{(1+q)}{\beta}\right) \int_{M} H^{2+2 q}\left\langle P_{1}(\nabla \varphi), \nabla \varphi\right\rangle d M .
\end{aligned}
$$

Let us estimate $\int_{M} H^{2 q} \varphi^{2}\left\langle P_{1}(\nabla H), \nabla H\right\rangle d M$. By using identity

$$
L_{1}(f g)=\operatorname{div}\left(P_{1}(\nabla(f g))\right)=\operatorname{div}\left(f P_{1}(\nabla g)\right)+g L_{1} f+\left\langle P_{1}(\nabla f), \nabla g\right\rangle \text {, }
$$


we have

$$
\begin{aligned}
L_{1}\left(H^{2+2 q} \varphi^{2}\right)= & \operatorname{div}\left(H P_{1}\left(\nabla\left(H^{1+2 q} \varphi^{2}\right)\right)\right)+H^{1+2 q} \varphi^{2} L_{1}(H) \\
& +\left\langle P_{1}(\nabla H), \nabla\left(H^{1+2 q} \varphi^{2}\right)\right\rangle \\
= & \operatorname{div}\left(H P_{1}\left(\nabla\left(H^{1+2 q} \varphi^{2}\right)\right)\right)+H^{1+2 q} \varphi^{2} L_{1}(H) \\
& +(1+2 q) H^{2 q} \varphi^{2}\left\langle P_{1}(\nabla H), \nabla H\right\rangle+2 H^{1+2 q} \varphi\left\langle P_{1}(\nabla H), \nabla \varphi\right\rangle .
\end{aligned}
$$

Integrating both sides of the identity above and by using Divergence Theorem, we obtain

$$
\begin{aligned}
(1+2 q) \int_{M} H^{2 q} \varphi^{2}\left\langle P_{1}(\nabla H), \nabla H\right\rangle d M= & -\int_{M} H^{1+2 q} \varphi^{2} L_{1}(H) d M \\
& -2 \int_{M} H^{1+2 q} \varphi\left\langle P_{1}(\nabla H), \nabla \varphi\right\rangle d M .
\end{aligned}
$$

By using inequality (3.3), we have

$$
\begin{aligned}
(1+2 q) \int_{M} H^{2 q} \varphi^{2}\left\langle P_{1}(\nabla H), \nabla H\right\rangle d M & \leq-\int_{M} H^{1+2 q} \varphi^{2} L_{1}(H) d M \\
& +\beta \int_{M} H^{2 q} \varphi^{2}\left\langle P_{1}(\nabla H), \nabla H\right\rangle \\
& +\frac{1}{\beta} \int_{M} H^{2+2 q}\left\langle P_{1}(\nabla \varphi), \nabla \varphi\right\rangle d M
\end{aligned}
$$

i.e.,

$$
\begin{aligned}
(1+2 q-\beta) \int_{M} H^{2 q} \varphi^{2}\left\langle P_{1}(\nabla H), \nabla H\right\rangle d M & \leq-\int_{M} H^{1+2 q} \varphi^{2} L_{1}(H) d M \\
& +\frac{1}{\beta} \int_{M} H^{2+2 q}\left\langle P_{1}(\nabla \varphi), \nabla \varphi\right\rangle d M .
\end{aligned}
$$

On the other hand, is well known, see [1], Lemma 3.7, that

$$
-L_{1}(H)=|\nabla H|^{2}-|\nabla A|^{2}-3 H K \text {. }
$$

Since $P_{1}$ is positive definite, we have

$$
\left\langle P_{1}(\nabla H), \nabla H\right\rangle \leq\left(\operatorname{tr} P_{1}\right)|\nabla H|^{2}=2 H|\nabla H|^{2},
$$


i.e.,

$$
|\nabla H|^{2} \geq \frac{1}{2 H}\left\langle P_{1}(\nabla H), \nabla H\right\rangle
$$

By using Proposition 2.1 and inequality above, we obtain

$$
-L_{1}(H) \leq-\frac{2}{1+2 c_{0}^{2}}|\nabla H|^{2}-3 H K \leq-\frac{1}{\left(1+2 c_{0}^{2}\right) H}\left\langle P_{1}(\nabla H), \nabla H\right\rangle-3 H K .
$$

Then

$$
\begin{aligned}
\left(1+\frac{1}{1+2 c_{0}^{2}}+2 q-\beta\right) \int_{M} H^{2 q} \varphi^{2}\left\langle P_{1}(\nabla H), \nabla H\right\rangle d M & \leq 3 \int_{M} H^{2+2 q}(-K) \varphi^{2} d M \\
& +\frac{1}{\beta} \int_{M} H^{2+2 q} \varphi\left\langle P_{1}(\nabla \varphi), \nabla \varphi\right\rangle d M
\end{aligned}
$$

Replacing last inequality in (3.4), stability inequality becomes

$$
\begin{aligned}
3 \int_{M}(-K) H^{2+2 q} \varphi^{2} d M \leq & 3 C_{1} \int_{M} H^{2+2 q} \varphi^{2}(-K) d M \\
& +C_{2} \int_{M} H^{2+2 q}\left\langle P_{1}(\nabla \varphi), \nabla \varphi\right\rangle d M
\end{aligned}
$$

i.e.,

$$
3\left(1-C_{1}\right) \int_{M} H^{2+2 q}(-K) \varphi^{2} d M \leq C_{2} \int_{M} H^{2+2 q}\left\langle P_{1}(\nabla \varphi), \nabla \varphi\right\rangle d M
$$

where

$$
C_{1}=\frac{(1+q)^{2}+\beta(1+q)}{1+\frac{1}{1+2 c_{0}^{2}}+2 q-\beta}, C_{2}=1+\frac{(1+q)}{\beta}+\frac{(1+q)^{2}+(1+q) \beta}{\beta\left(1+\frac{1}{1+2 c_{0}^{2}}+2 q-\beta\right)}
$$

$0<q<\sqrt{\frac{1}{1+2 c_{0}^{2}}}$ by hypothesis, and $\beta$ is taken such that $0<\beta<\frac{\frac{1}{1+2 c_{0}^{2}}-q^{2}}{q+2}$. This choice of $\beta$ is necessary to have $C_{1}<1$. In fact,

$$
\begin{aligned}
\beta<\frac{\frac{1}{1+2 c_{0}^{2}}-q^{2}}{q+2} & \Rightarrow q^{2}+\beta q+2 \beta<\frac{1}{1+2 c_{0}^{2}} \\
& \Rightarrow(1+q)^{2}+\beta(1+q)<1+\frac{1}{1+2 c_{0}^{2}}+2 q-\beta \\
& \Rightarrow C_{1}=\frac{(1+q)^{2}+\beta(1+q)}{1+\frac{1}{1+2 c_{0}^{2}}+2 q-\beta}<1 .
\end{aligned}
$$


Therefore,

$$
\int_{M} H^{2+2 q}(-K) \varphi^{2} d M \leq \frac{C_{2}}{3\left(1-C_{1}\right)} \int_{M} H^{2+2 q}\left\langle P_{1}(\nabla \varphi), \nabla \varphi\right\rangle d M
$$

On the other hand, since $P_{1}$ is positive definite, we have

$$
\left\langle P_{1}(\nabla \varphi), \nabla \varphi\right\rangle \leq\left(\operatorname{tr} P_{1}\right)|\nabla H|^{2} \leq 2 H|\nabla \varphi|^{2} .
$$

Denoting by $C_{3}=\frac{2 C_{2}}{3\left(1-C_{1}\right)}$, we have

$$
\begin{aligned}
\int_{M} H^{2+2 q}(-K) \varphi^{2} d M & \leq \frac{C_{3}}{2} \int_{M} H^{2+2 q}\left\langle P_{1}(\nabla \varphi), \nabla \varphi\right\rangle d M \\
& \leq C_{3} \int_{M} H^{3+2 q}|\nabla \varphi|^{2} d M
\end{aligned}
$$

Letting $\varphi=\psi^{p}$, where $2 p=5+2 q$, we obtain

$$
\int_{M} H^{2+2 q}(-K) \psi^{5+2 q} d M \leq C_{3} p^{2} \int_{M} H^{3+2 q} \psi^{3+2 q}|\nabla \psi|^{2} d M .
$$

By using Young's inequality, i.e.,

$$
x y \leq \frac{x^{a}}{a}+\frac{y^{b}}{b}, \frac{1}{a}+\frac{1}{b}=1
$$

with

$$
x=\delta H^{3+2 q} \psi^{3+2 q}, \quad y=\frac{|\nabla \psi|^{2}}{\delta}, \quad a=\frac{5+2 q}{3+2 q}, \quad b=\frac{5+2 q}{2}, \quad \text { and } \delta>0,
$$

we have

$$
H^{3+2 q} \psi^{3+2 q}|\nabla \psi|^{2} \leq \frac{3+2 q}{5+2 q} \delta^{\frac{5+2 q}{3+2 q}} H^{5+2 q} \psi^{5+2 q}+\frac{2}{5+2 q} \delta^{-\frac{5+2 q}{2}}|\nabla \psi|^{5+2 q} .
$$

Replacing last inequality in inequality (3.5), we obtain

$$
\begin{aligned}
\int_{M} H^{2+2 q}(-K) \psi^{5+2 q} d M \leq & \frac{3+2 q}{5+2 q} p^{2} C_{3} \delta^{\frac{5+2 q}{3+2 q}} \int_{M} H^{5+2 q} \psi^{5+2 q} d M \\
& +\frac{2}{5+2 q} p^{2} C_{3} \delta^{-\frac{5+2 q}{2}} \int_{M}|\nabla \psi|^{5+2 q} d M
\end{aligned}
$$


i.e.,

$$
\int_{M} H^{5+2 q}\left(\frac{(-K)}{H^{3}}-\Lambda_{1} \delta^{\frac{5+2 q}{3+2 q}}\right) \psi^{5+2 q} d M \leq \Lambda_{2} \delta^{-\frac{5+2 q}{2}} \int_{M}|\nabla \psi|^{5+2 q} d M,
$$

where $\Lambda_{1}=\frac{3+2 q}{5+2 q} p^{2} C_{3}$ and $\Lambda_{2}=\frac{2 p^{2}}{5+2 q} C_{3}$.

Remark 3.1. In [15], Schoen, Simon, and Yau obtained the following Sobolev type inequality for minimal hypersurfaces $M^{n}$ immersed in $\mathbb{R}^{n+1}$ :

$$
\int_{M}|A|^{2 p} \psi^{2 p} d M \leq C(n, p) \int_{M}|\nabla \phi|^{2 p} d M
$$

for $p \in[2,2+\sqrt{2 / n})$, and for all function $\psi: M \rightarrow \mathbb{R}$ compactly supported on $M$. By using inequality of Proposition 3.1, we obtain a similar result for hypersurfaces $M^{3}$ immersed in $\mathbb{R}^{4}$ with zero scalar curvature. In fact, if $R=0$, then $H^{2}=|A|^{2}$. Choosing an orientation such that $H>0$, we have $H=|A|$. In this case, we have

Corollary 3.1 (Sobolev type inequality). Let $x: M^{3} \rightarrow \mathbb{R}^{4}$ be a stable isometric immersion with zero scalar curvature and such that $\frac{(-K)}{H^{3}} \geq c>0$ everywhere. Then, for all smooth function $\psi$ with compact support in $M$, for all $\delta>0$ and $p \in\left(\frac{5}{2}, \frac{5}{2}+\sqrt{\frac{1}{1+2 c_{0}^{2}}}\right)$, there exists a constant $C(p)>0$ such that

$$
\int_{M}|A|^{2 p} \psi^{2 p} d M \leq C(p) \int_{M}|\nabla \psi|^{2 p} d M
$$

Remark 3.2. In the recent article [11], Ilias, Nelli, and Soret, obtained results in this direction for hypersurfaces with constant mean curvature.

Now let us prove the Theorem A stated in the Introduction.

Theorem A. There is no stable complete hypersurface $M^{3}$ of $\mathbb{R}^{4}$ with zero scalar curvature, polynomial volume growth and such that

$$
\frac{(-K)}{H^{3}} \geq c>0
$$

everywhere, for some constant $c>0$. 
Proof. Suppose by contradiction there exists a complete stable hypersurface attending conditions of Theorem A. Then we can apply Proposition 3.1. Choose the compact supported function $\psi: M \rightarrow \mathbb{R}$ defined by

$$
\psi(\rho(p))=\left\{\begin{array}{cl}
1 & \text { if } p \in B_{r} ; \\
\frac{2 r-\rho(p)}{r} & \text { if } p \in B_{2 r} \backslash B_{r} ; \\
0 & \text { if } p \in M \backslash B_{2 r},
\end{array}\right.
$$

where $\rho(p)=\rho\left(p, p_{0}\right)$ is the distance function of $M$. By using this function $\psi$ in the inequality of Proposition 3.1, we have

$$
\begin{aligned}
\int_{B_{r}} H^{5+2 q}\left(\frac{(-K)}{H^{3}}-\Lambda_{1} \delta^{\frac{5+2 q}{3+2 q}}\right) d M & \leq \int_{B_{2 r}} H^{5+2 q}\left(\frac{(-K)}{H^{3}}-\Lambda_{1} \delta^{\frac{5+2 q}{3+2 q}}\right) \psi^{5+2 q} d M \\
& \leq \Lambda_{2} \delta^{-\frac{5+2 q}{2}} \int_{B_{2 r}}|\nabla \psi|^{5+2 q} d M \\
& \leq \Lambda_{2} \delta^{-\frac{5+2 q}{2}} \frac{\mathrm{vol} B_{2 r}}{r^{5+2 q}}
\end{aligned}
$$

for $0<q<\sqrt{\frac{1}{1+2 c_{0}^{2}}}$. Taking $\delta>0$ sufficiently small and since, by hypothesis, $\frac{(-K)}{H^{3}} \geq$ $c>0$, we get

$$
\left(\frac{(-K)}{H^{3}}-\Lambda_{1} \delta^{\frac{5+2 q}{3+2 q}}\right)>0 .
$$

By hypothesis, $M$ has polynomial volume growth. It implies that

$$
\lim _{r \rightarrow \infty} \frac{\operatorname{vol}\left(B_{r}\right)}{r^{\alpha}}<\infty, \alpha \in(0,4] .
$$

Letting $r \rightarrow \infty$ in the inequality (3.10), we obtain

$$
\lim _{r \rightarrow \infty} \int_{B_{r}} H^{5+2 q}\left(\frac{(-K)}{H^{3}}-\Lambda_{1} \delta^{\frac{5+2 q}{3+2 q}}\right) d M \leq \Lambda_{2} \lim _{r \rightarrow \infty} \frac{\operatorname{vol}\left(B_{2 r}\right)}{r^{\alpha}} \cdot \lim _{r \rightarrow \infty} \frac{1}{r^{5+2 q-\alpha}}=0 .
$$

Therefore $H \equiv 0$, and this contradiction finishes the proof of the theorem.

Remark 3.3. In the proof of Theorem A, $M$ need not even be properly immersed, since we are taking intrinsic (geodesic) balls. Since $M$ is complete, we have $M=\bigcup_{n=1}^{\infty} B_{r_{n}}$ for some sequence $r_{n} \rightarrow \infty$, and thus we can take $r \rightarrow \infty$ in the estimate. 
Remark 3.4. By using their Sobolev inequality (3.7), Schoen, Simon, and Yau gave a new proof of Bernstein's Theorem for dimension less than or equal to 5, namely, that the only entire minimal graphs $M^{n}$ in $\mathbb{R}^{n+1}, n \leq 5$ are hyperplanes. By using our version of Sobolev inequality (3.8), we prove the following Bernstein type result.

As a corollary of Theorem A, we have the following result.

Theorem B. There are no entire graphs $M^{3}$ of $\mathbb{R}^{4}$ with zero scalar curvature and such that

$$
\frac{(-K)}{H^{3}} \geq c>0
$$

everywhere, for some constant $c>0$.

Proof. Suppose there exists an entire graph $M$ satisfying the conditions of corollary. In [3], Proposition 4.1, p. 3308, Alencar, Santos, and Zhou showed that entire graphs with zero scalar curvature and whose mean curvature does not change sign are stable. Since $R=0$ by hypothesis, we have $H^{2}=|A|^{2}$. Provided $K \neq 0$ everywhere, we have $H^{2}=|A|^{2}>0$ which implies that $H$ does not change sign. Thus, the entire graph $M$ is stable. On the other hand, is well known that graphs satisfies $\operatorname{vol}\left(B_{r}\right) \leq C r^{4}, C>0$. Therefore, by using the hypothesis $\frac{-K}{H^{3}} \geq c>0$, inequality 3.10 , in the proof of Theorem A, p.16, and taking $r \rightarrow \infty$ we obtain the same contradiction.

\section{Examples}

The class of hypersurfaces treated here is non-empty, as shown in the following example. It can be found in [10], Lemma 2.1, p. 400. See also [2], p. $213-214$ and [7], p. 161.

Example 4.1. Let $M^{3} \hookrightarrow \mathbb{R}^{4}$ the rotational hypersurface parametrized by

$$
X(t, \theta, \varphi)=(f(t) \operatorname{sen} \theta \cos \varphi, f(t) \operatorname{sen} \theta \operatorname{sen} \varphi, f(t) \cos \theta, t)
$$

where $f(t)=\frac{t^{2}}{4 m}+m$ and $m$ is a non-negative constant. The principal curvatures are

$$
\lambda_{1}=\lambda_{2}=\frac{m^{1 / 2}}{f^{3 / 2}}, \lambda_{3}=-\frac{1}{2} \frac{m^{1 / 2}}{f^{3 / 2}} .
$$


Then $R=0$ and $\frac{-K}{H^{3}}=\frac{4}{27}$ everywhere. Since $M^{3}$ is a rotational hypersurface and its profile curve is quadratic, it has polynomial volume growth. Then by Theorem A the immersion is unstable.

This example appears in the Theory of Relativity as the embedding of the space-like Schwarzschild manifold of mass $m / 2>0$, see Introduction of [5], for details.

The following class of hypersurfaces are well known, see [2], p. 214, and they are the classical examples of stable hypersurfaces with zero scalar curvature. This class show us that some condition over nullity of Gauss-Kronecker curvature are needed.

Example 4.2. Let $M^{3} \subset \mathbb{R}^{4}$ be the cylinder parametrized by

$$
\mathbf{x}(u, v, t)=(u, v, \alpha(t), \beta(t)), u, v, t \in \mathbb{R}
$$

where $c(t):=(\alpha(t), \beta(t))$ is a parametrized curve with positive curvature $k(t)$ at every point. In this case, principal curvatures are

$$
\lambda_{1}=0, \lambda_{2}=0, \text { and } \lambda_{3}=k(t)
$$

Thus $R=0, H>0$ and $K=0$ everywhere. Then, $M^{3}$ is stable, see [2].

Observe that if $c(t)=(t, f(t))$, the cylinder $M$ is the graph of the smooth function $F: \mathbb{R}^{3} \rightarrow \mathbb{R}$ given by $F(u, v, t)=f(t)$. In particular, taking $f(t)=t^{2}$ or $f(t)=\sqrt{1+t^{2}}$ we obtain an entire graph with polynomial volume growth, $R=0, H>0$ and $K=0$ everywhere.

\section{Non-embedded Tubes}

Let $x: M^{3} \rightarrow \mathbb{R}^{4}$ be an isometric immersion. Following Nelli and Soret, see [12], we define the tube of radius $h$ around $M$ the set

$$
T(M, h)=\left\{x \in \mathbb{R}^{4} ; \exists p \in M, x=p+t \eta, t \leq h(p)\right\}
$$


where $\eta$ is the normal vector of the second fundamental form of $x$, and $h: M \rightarrow \mathbb{R}$ is an everywhere non-zero smooth function. If $|A| \neq 0$ everywhere, we define the subfocal tube the set

$$
T\left(M, \frac{\epsilon}{|A|}\right), 0<\epsilon \leq 1
$$

Denote by $T(r, h)$ the tube of radius $h$ around $B_{r} \subset M$, i.e., considering $M=B_{r}$ in the above definition, and let

$$
V(r, h)=\int_{T(r, h)} d T
$$

where $d T$ denotes the volume element of the tube. If $R=0$, and choosing an orientation such that $H>0$, we have $H=|A|$. Under the conditions of Proposition 3.1, and assuming that $\frac{(-K)}{H^{3}} \geq c>0$, then there exists a constant $C(q)$ depending only on $0<q<\sqrt{\frac{1}{1+2 c_{0}^{2}}}$ such that

$$
\int_{B_{r}}|A|^{5+2 q} \psi^{5+2 q} d M \leq C(q) \int_{B_{r}}|\nabla \psi|^{5+2 q} d M .
$$

Choosing the same function with compact support used in the proof of Theorem A (see (3.9), p. 16), we obtain

$$
\int_{B_{r}}|A|^{5+2 q} d M \leq C(q) \frac{\operatorname{vol}\left(B_{r}\right)}{r^{5+2 q}} .
$$

The following lemma is essentially the same Lemma 1 of [12], p. 496, and the proof will be omitted here.

Lemma 5.1. Let $M^{3}$ be a complete, stable hypersurface of $\mathbb{R}^{4}$ satisfying $R=0$ and $\frac{(-K)}{H^{3}} \geq c>0$ everywhere.

(a) For $r>0$ sufficiently large, there exists a constant $\alpha(q)$, depending only on $0<q<$ $\sqrt{\frac{1}{1+2 c_{0}^{2}}}$ such that

$$
\operatorname{vol}\left(B_{r}\right)>\alpha(q) r^{5+2 q}
$$

(b) For each $\beta>1,0<q<\sqrt{\frac{1}{1+2 c_{0}^{2}}}$, and $r>0$ satisfying inequality 5.2 above, there exists a sufficiently large $\tilde{r}>r$ such that

$$
\operatorname{vol}\left(B_{\tilde{r}}\right)-\operatorname{vol}\left(B_{\beta^{-1}} \tilde{r}\right)>\alpha(q) r^{5+q}
$$


The next result is a vanishing scalar curvature version of Theorem 1, p. 499 of [12].

Theorem C. Let $M^{3}$ be a stable complete hypersurface of $\mathbb{R}^{4}$ with vanishing scalar curvature. Suppose that the second fundamental form of the immersion is bounded and there exists a constant $c>0$ such that $\frac{(-K)}{H^{3}} \geq c>0$ everywhere. Then, for constants $0<b_{1} \leq 1, b_{2}>0$, and for any smooth function $h: M \rightarrow \mathbb{R}$ satisfying

$$
h(p) \geq \inf \left\{\frac{b_{1}}{|A(p)|}, b_{2} \rho(p)^{\delta}\right\}, \delta>0,
$$

the tube $T(M, h)$ is not embedded. Here, $\rho(p)$ denotes the intrinsic distance in $M$ to a fixed point $p_{0} \in M$.

Proof. In [12], Nelli and Soret showed that

$$
V(r, h)=\int_{B_{r}} h(p) d M-\frac{1}{2} \int_{B_{r}} h(p)^{2} H(p) d M-\frac{1}{4} \int_{B_{r}} h(p)^{4} K(p) d M .
$$

By using the classical inequality between geometric and quadratic means, one finds that

$$
\begin{aligned}
K & =\lambda_{1} \lambda_{2} \lambda_{3} \leq\left|\lambda_{1}\right|\left|\lambda_{2}\right|\left|\lambda_{3}\right| \\
& \leq\left(\frac{\lambda_{1}^{2}+\lambda_{2}^{2}+\lambda_{3}^{2}}{3}\right)^{3 / 2} \\
& =\frac{1}{3 \sqrt{3}}|A|^{3}
\end{aligned}
$$

i.e.,

$$
K(p) \leq \frac{1}{3 \sqrt{3}}|A(p)|^{3}
$$

Let $B_{r}^{+}$the set where $\frac{b_{1}}{|A(p)|}$ is the infimum and $B_{r}^{-}=B_{r} \backslash B_{r}^{+}$. Then

$$
\begin{aligned}
V(r, h) \geq & b_{1} \int_{B_{r}^{+}} \frac{1}{|A|} d M-\frac{b_{1}^{2}}{2} \int_{B_{r}^{+}} \frac{1}{|A|^{2}} H d M-\frac{b_{1}^{4}}{4} \int_{B_{r}^{+}} \frac{1}{|A|^{4}} K d M \\
& +b_{2} \int_{B_{r}^{-}} \rho^{\delta} d M-\frac{b_{2}^{2}}{2} \int_{B_{r}^{-}} \rho^{2 \delta} H d M-\frac{b_{2}^{4}}{4} \int_{B_{r}^{-}} \rho^{4 \delta} K d M .
\end{aligned}
$$


Since $H=|A|$, we have

$$
\begin{aligned}
V(r, h) \geq & \left(b_{1}-\frac{b_{1}^{2}}{2}-\frac{b_{1}^{4}}{12 \sqrt{3}}\right) \int_{B_{r}^{+}} \frac{1}{|A|} d M \\
& +b_{2} \int_{B_{r}^{-}} \rho^{\delta} d M-\frac{b_{2}^{2}}{2} \int_{B_{r}^{-}} \rho^{2 \delta} H d M-\frac{b_{2}^{4}}{4} \int_{B_{r}^{-}} \rho^{4 \delta} K d M .
\end{aligned}
$$

Let us estimate the integrals over $B_{r}^{-}$. By using inequality (5.4) above, we get

$$
-K \geq-\frac{1}{3 \sqrt{3}}|A|^{3} \geq-\frac{b_{1}}{b_{2}} \frac{1}{3 \sqrt{3}} \rho^{-3 \delta}
$$

and

$$
-H=-|A| \geq \frac{b_{1}}{b_{2}} \rho^{-\delta}
$$

By hypothesis, $|A|$ is bounded, then there exists $a:=\inf _{M} \frac{1}{|A|}$. Therefore

$$
\begin{aligned}
V(r, h) & \geq\left(b_{1}-\frac{b_{1}^{2}}{2}-\frac{b_{1}^{4}}{12 \sqrt{3}}\right) \int_{B_{r}^{+}} \frac{1}{|A|} d M+\left(b_{2}-\frac{b_{2} b_{1}}{2}-\frac{b_{2}^{3} b_{1}}{12 \sqrt{3}}\right) \int_{B_{r}^{-}} \rho^{\delta} d M \\
& \geq a\left(b_{1}-\frac{b_{1}^{2}}{2}-\frac{b_{1}^{4}}{12 \sqrt{3}}\right) \operatorname{vol}\left(B_{r}^{+}\right)+\left(b_{2}-\frac{b_{2} b_{1}}{2}-\frac{b_{2}^{3} b_{1}}{12 \sqrt{3}}\right) \int_{B_{r}^{-}} \rho^{\delta} d M .
\end{aligned}
$$

On the other hand, for $r$ sufficiently large,

$$
\begin{aligned}
\int_{B_{r}^{-}} \rho^{\delta} d M & =\int_{B_{r}^{-} \backslash B_{\beta^{-1} 1_{r}}^{-}} \rho^{\delta} d M+\int_{B_{\beta^{-1} r_{r}}^{-}} \rho^{\delta} d M \geq \int_{B_{r}^{-} \backslash B_{\beta^{-1} 1_{r}}^{-}} \rho^{\delta} d M \\
& \geq\left(\frac{r}{\beta}\right)^{\delta}\left[\operatorname{vol}\left(B_{r}^{-}\right)-\operatorname{vol}\left(B_{\beta^{-1} r}^{-}\right)\right] \\
& \geq\left[\operatorname{vol}\left(B_{r}^{-}\right)-\operatorname{vol}\left(B_{\beta^{-1} r}^{-}\right)\right] .
\end{aligned}
$$


Then

$$
\begin{aligned}
V(r, h) \geq & a\left(b_{1}-\frac{b_{1}^{2}}{2}-\frac{b_{1}^{4}}{12 \sqrt{3}}\right)\left(\operatorname{vol}\left(B_{r}^{+}\right)-\operatorname{vol}\left(B_{\beta^{-1} r}^{+}\right)+\operatorname{vol}\left(B_{\beta^{-1} r}^{+}\right)\right) \\
& +\left(b_{2}-\frac{b_{2}^{2} b_{1}}{2}-\frac{b_{2}^{3} b_{1}}{12 \sqrt{3}}\right)\left(\operatorname{vol}\left(B_{r}^{-}\right)-\operatorname{vol}\left(B_{\beta^{-1} r}^{-}\right)\right) \\
\geq & C\left[\operatorname{vol}\left(B_{r}\right)-\operatorname{vol}\left(B_{\beta^{-1} r}\right)\right] .
\end{aligned}
$$

By using Lemma 5.1, item $(b)$, there exists $\tilde{r}>r$ such that

$$
V(\tilde{r}, h) \geq C \tilde{r}^{5+q}
$$

The Euclidean distance is less than or equal to the intrinsic distance. It implies

$$
B_{r}(p) \subset \mathbb{B}(p, r)
$$

where $B_{r}(p) \equiv B_{r}$ and $\mathbb{B}(p, r)$ denotes the intrinsic and the Euclidean ball of center $p$ and radius $r$. By using (5.3), we have

$$
h(q) \geq \min \left\{\frac{b_{1}}{|A|}, b_{2} \rho(q)^{\delta}\right\} \geq \min \left\{\inf _{M} \frac{b_{1}}{|A|}, b_{2} \rho(q)^{\delta}\right\}=\inf _{M} \frac{b_{1}}{|A|}=b_{1} a,
$$

for $0<b_{1} \leq 1$ and $\rho$ sufficiently large, then

$$
T\left(r, b_{1} a\right) \subset T(r, h) .
$$

Suppose, by contradiction, that $T\left(r, b_{1} a\right)$ is embedded. Since

$$
T\left(r, b_{1} a\right) \subset \mathbb{B}\left(p, r+2 b_{1} a\right)
$$

then its volume $V\left(r, b_{1} a\right)$ satisfies

$$
V\left(r, b_{1} a\right) \leq \operatorname{vol}\left(\mathbb{B}\left(p, r+2 b_{1} a\right)\right)=\omega_{4}\left(r+2 b_{1} a\right)^{4},
$$

where $\omega_{4}$ is the volume of $\mathbb{B}(p, 1)$. Let us consider two different cases. First, if $M$ is not contained in any ball, above inequality is a contradiction with (5.5) for $r$ sufficiently large. Therefore, $T\left(r, b_{1} a\right)$, and thus $T(r, h)$, is not embedded for $r$ sufficiently large. In the second case, if $M$ is contained in some ball, then $T(M, h)$ has finite volume (since $T(M, h)$ is embedded) and it is also a contradiction with 5.5 . 


\section{Appendix}

Let us prove the following fact established in the Introduction:

Let $x: M^{3} \rightarrow \mathbb{R}^{4}$ be an isometric immersion with zero scalar curvature. If $H$ and $K$ denotes the mean curvature and Gauss-Kronecker curvature, respectively, then

$$
0 \leq \frac{-K}{H^{3}} \leq \frac{4}{27} \text { everywhere on } M
$$

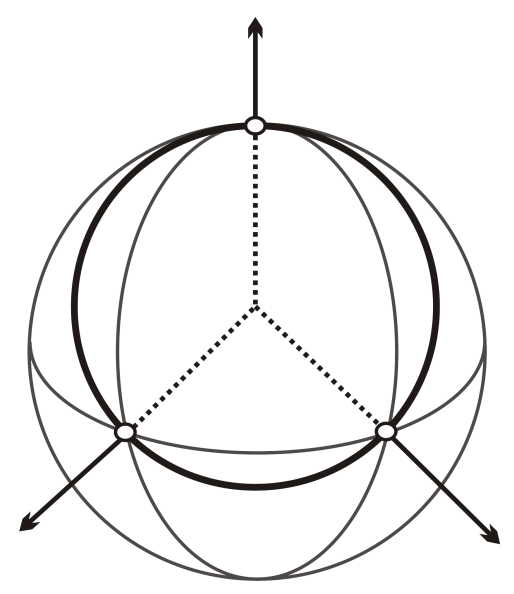

Figure 1: Representation of the domain $N_{\omega}$ of $\frac{K}{H^{3}}$ over $\mathbb{S}^{2}$, considering this function as an algebraic function of the eigenvalues. This domain is the intersection of one of the plane $\lambda_{1}+\lambda_{2}+\lambda_{3}=1$ with $\mathbb{S}^{2}$. The hypothesis cuts off only three small neighbourhoods around the coordinate axis.

In fact, let $\left(\lambda_{1}, \lambda_{2}, \lambda_{3}\right)=t \omega$ where $\omega \in \mathbb{S}^{2}$. By using (1.1), we can see that $R, H$ and $K$ are homogeneous polynomials. It implies $H(t \omega)=t H(\omega), R(t \omega)=t^{2} R(\omega), K(t \omega)=$ $t^{3} K(\omega)$ and hence

$$
\frac{K}{H^{3}}(t \omega)=\frac{K}{H^{3}}(\omega)
$$

Then the behavior of $\frac{K}{H^{3}}$ depends only of its values on the sphere $\mathbb{S}^{2}$. Since $N:=$ $\left\{\left(\lambda_{1}, \lambda_{2}, \lambda_{3}\right) \in \mathbb{R}^{3} ; R=\lambda_{1} \lambda_{2}+\lambda_{1} \lambda_{3}+\lambda_{2} \lambda_{3}=0\right\}$ is closed and $\mathbb{S}^{2}$ is compact, we obtain that $N_{\omega}=N \cap \mathbb{S}^{2}$ is compact, see figure 6. Then, $\frac{K}{H^{3}}: N_{\omega} \rightarrow \mathbb{R}$ is a continuous 
function with compact domain. The claim then follow from the Weierstrass maxima and minima theorem. Upper bound $\frac{4}{27}$ can be found by using Lagrange multipliers method.

\section{References}

[1] Alencar, H., do Carmo, M. P., Colares, A. G., Stable hypersurfaces with constant scalar curvature, Math. Z., 213, 117 - 131 (1993).

[2] Alencar, H., do Carmo, M. P., Elbert, M. F., Stability of hypersurfaces with vanishing $r$-constant curvatures in Euclidean spaces. J. Reine Angew. Math. 554, 201-216 (2003).

[3] Alencar, H., Santos W., Zhou W., Stable hypersurfaces with constant scalar curvature, Proc. Amer. Math. Soc., 138, 3301 - 3312 (2010).

[4] Barbosa, J.L., Colares, A.G., Stability of hypersurfaces with constant $r$-mean curvature. Annals of Global Analysis and Geometry, 15, 277-297 (1997).

[5] Bray, H. L., Proof of the Riemannian Penrose inequality using the positive mass theorem, J. Diff. Geo., 59, 177-267 (2001).

[6] Do Carmo, M.; Peng, C. K. Stable complete minimal hypersurfaces. Proceedings of the 1980 Beijing Symposium on Differential Geometry and Differential Equations, Vol. 1, 2, 3 (Beijing, 1980), 1349-1358, Sci. Press Beijing, Beijing, (1982).

[7] Do Carmo, M., Elbert M. F., On Stable complete Hypersurfaces with vanishing r-mean curvature, Tohoku Math. J., 56, 155-162 (2004).

[8] Hartman, P., Nirenberg, L., On spherical image maps whose Jacobians do not change sign. Amer. J. Math. 81, 901-920 (1959).

[9] Hounie, J., Leite, M. L., Two-ended hypersurfaces with zero scalar curvature, Indiana University Mathematical Journal, 48, 867-882 (1999). 
[10] Hounie, J., Leite, M. L., Uniqueness and nonexistence theorems for hypersurfaces with $H_{r}=0$, Annals of Global Analysis and Geometry, 17, 397-407 (1999).

[11] Ilias, S., Nelli, B., Soret, M., Caccioppoli's inequalities on constant mean curvature hypersurfaces in Riemannian manifolds, Annals of Global Analysis and Geometry, 42, no. 4, 433-471 (2012).

[12] Nelli, B., Soret, M., Stably embedded minimal hypersurfaces. Mathematische Zeitschrift. 255, 493-514 (2007).

[13] Reilly, R. C., Variational properties of functions of the mean curvatures for hypersurfaces in space forms, J. Diff. Geometry. 8, 465-477 (1973).

[14] Rosenberg, H., Hypersurfaces of constant curvatures in space forms. Bulletin des Sciences Mathématiques, 117, 211-239 (1993).

[15] Schoen, R., Simon, L., Yau, S-T. Curvature estimates for minimal hypersurfaces, Acta Math. 134, 275 - 288 (1975).

[16] Cheng, S.Y., Yau, S.T., Hypersurfaces with constant scalar curvature. Math. Ann. 225, 195-204 (1977).

Gregório Silva Neto

Universidade Federal de Alagoas,

Instituto de Matemática,

57072-900, Maceió, Alagoas, Brazil.

gregorio@im.ufal.br 